\title{
Ballistic resistivity in aluminum nanocontacts
}

\author{
A. Hasmy, ${ }^{1}$ A. J. Pérez-Jiménez, ${ }^{2}$ J. J. Palacios, ${ }^{2}$ P. García-Mochales, ${ }^{3}$ J. L. Costa-Krämer, ${ }^{4}$ M. Díaz, ${ }^{1}$ \\ E. Medina, ${ }^{1}$ and P. A. Serena ${ }^{3}$ \\ ${ }^{1}$ Centro de Física, IVIC, Apdo. 21827, Caracas 1020A, Venezuela \\ ${ }^{2}$ Departamento de Física Aplicada, Universidad de Alicante, San Vicente del Raspeig, 03690-Alicante, Spain \\ ${ }^{3}$ Instituto de Ciencia de Materiales de Madrid, CSIC, Cantoblanco, 28049-Madrid, Spain \\ ${ }^{4}$ Instituto de Microelectrónica de Madrid, CSIC, Isaac Newton 8, PTM, 28760-Tres Cantos, Madrid, Spain
}

(Received 20 October 2004; published 5 December 2005)

\begin{abstract}
We perform a representative series of semiclassical molecular dynamics simulations of aluminum nanocontact breakages, coupled to full quantum conductance calculations. This approach allows to obtain realistic conductance histograms of polyvalent species and understand the origin of their peaked structures. The results show that the conductance depends linearly on the contact minimum cross section for the geometrically favored nanocontact configurations. Valid in a broad range of conductance values, such relation suggests the definition of a transport parameter for the nanoscale, that represents the novel concept of ballistic resistivity.
\end{abstract}

DOI: $10.1103 /$ PhysRevB.72.245405

PACS number(s): 73.23.Ad, 73.40.Jn

One of the major industrial challenges is to profit from some fascinating physical features present at the nanoscale. The production of dissipationless nanoswitches (or nanocontacts) is one of such attractive applications. ${ }^{1}$ The inelastic electron mean free path is usually larger than nanocontact typical cross sections (of the order of few atoms in controlled experimets) even at room temperature, and, therefore, the electronic transport through these nanoconstrictions is expected to be ballistic. Nevertheless, the lack of knowledge of the real efficiency of this electronic ballistic/nondissipative transport limits future innovations.

For contact sizes of the order of a few Fermi wavelengths $\lambda_{F}$, well defined modes (channels) appear associated with the transversal confinement of electrons. For this situation, the conductance $G$ is given by the Landauer formula $G$ $=G_{0} \sum_{n=1}^{N} T_{n}$, where $G_{0}=2 \mathrm{e}^{2} / \mathrm{h}$ is the conductance quantum (e being the electron charge and $\mathrm{h}$ Planck's constant), $T_{n}$ is the transmission probability of the $n$th channel, and $N$ is the number of propagating modes with energies below the Fermi energy. $^{2}$ It has been shown that the number of conducting channels is determined by the number of valence electrons of the respective chemical element. ${ }^{3}$ For monovalent noble metals such as $\mathrm{Cu}, \mathrm{Ag}$ and $\mathrm{Au}$, the transmission probability $T$ has been estimated to be approximately equal to 1 (i.e., each noble-metal atom contact contributes with $G_{0}$ to the conductance value $\left.{ }^{4,5}\right)$. But for monovalent alkali metals or polyvalent chemical species, single-atom contact studies revealed that this channel transmittivity can have a result smaller than one. $^{3,6,7}$

Nowadays, there exist several experimental techniques to characterize the electronic transport through nanocontacts. Among them, the measurement of the conductance histogram during nanocontact breakages ${ }^{8-10}$ is one of the most used. By putting in contact two opposite electrodes and then separating them, one observes a stepwise decrease in the electrical conductance (i.e., a conductance scan), until the breakpoint is reached. ${ }^{11-14}$ It has been shown that each scan of the conductance dependence on the electrode retraction differs from one another, since the nanocontact structural evolutions during breakages are not identical. ${ }^{15}$ Notwith- standing for fixed experimental parameter conditions (such as temperature and applied voltage), the accumulation of data from many scans gives rise to a conductance histogram with a well reproducible peaked structure. The histogram peaks have been associated with preferred geometrical neck configurations during the nanocontact breakages. ${ }^{9,10,16} \mathrm{Nev}-$ ertheless, a consensed relation between the conductance and the contact size is lacking in the literature. ${ }^{17-20}$ On one hand, many theoretical studies have neglected the statistical feature of the experiments. On the other hand, almost all of these studies were based on semiclassical approximations, ${ }^{17-19}$ which tend to fail at the atomic scale because they do not describe the quantum character of the scattering process. ${ }^{2}$ Besides, in a contact of few atoms it is not clear how to express the number of atoms in terms of the contact size (i.e., the cross-sectional area ${ }^{19,20}$ ).

Although there exist powerful ab initio codes able to describe both, the atomic motion and the electron transport, on a full quantum mechanics basis, nowadays it is unfeasible to use them to construct and understand theoretical conductance histograms since they involve huge computational resources. In order to overcome the existing difficulties in this paper we have implemented an approach where the state-of-the-art embedded atom molecular dynamics method for the simulations of aluminum nanocontact ruptures ${ }^{10,15,21}$ has been coupled with full quantum calculations of the electron transport using a procedure based on the $a b$ initio Gaussian embedded-cluster method. ${ }^{22}$ The results reveal a statistically linear relationship between the conductance and the number of aluminum atoms contact. A similar strategy has been recently proposed ${ }^{23}$ for constructing computational gold conductance histograms, although a parametrized tight-binding approach has been used to calculate conductance values.

For the aluminum nanocontact simulations, we considered a similar procedure as introduced elsewhere, ${ }^{15}$ in which 1008 aluminum atoms were initially distributed in a supercell formed by 18 layers perpendicular to the (111) fcc direction, containing 56 atoms each. The lattice constant is initially taken to be $4.05 \AA$. The direction (111) corresponds to that in which the contact is elongated until breakdown. Simulations 


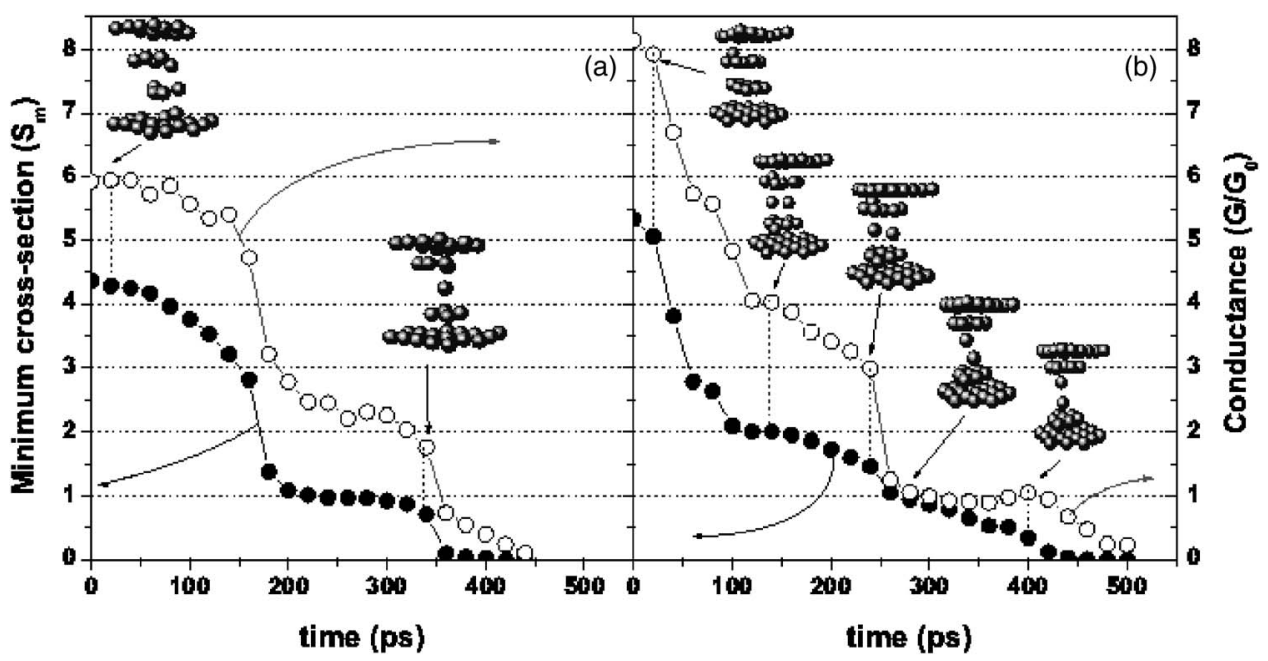

FIG. 1. Time evolution of the minimum cross section (black symbols) and the quantum conductance (open symbols) corresponding to two aluminum wire breakages at $T=4 \mathrm{~K}$, illustrating two rupture mechanisms: (a) the formation of a single-central atomic configuration before breakage, and (b) the formation of a dimer-chain contact before breakage. Insets depict several atomic configurations during nanowire stretching. Arrows and dashed vertical lines denote the corresponding conductance and minimum cross section associated with these nanoneck configurations.

are performed at $4 \mathrm{~K}$. In a first stage the system is relaxed during 50 ps. After this relaxation, two bilayer slabs are defined at the top and bottom of the relaxed supercell, and are separated. We have considered a stretching velocity $(2 \mathrm{~m} / \mathrm{s})$ much bigger than in typical nanocontact experiments, but it is still small compared to the speed of sound through the material (i.e., there is enough time for the system relaxation). The atoms inside these slabs are frozen during subsequent stages, defining the bulk supports of the nanocontact during the breaking process. The other atoms move and reaccommodate into new configurations during the elongation process. The full determination of atomic positions during contact stretching allows the evaluation of the evolution of its minimum cross-section $S_{m}$. The determination of $S_{m}$ (in units of number of atoms) has been done following standard numerical procedures. ${ }^{19}$ This method has been previously used to construct minimum cross-section histograms ${ }^{10,15}$ able to determine the important role deserved for favorable atomic configurations during the breakage process.

Pursuing a similar strategy to that of nanocontact transport experiments, we performed many numerical realizations of wire breakages for the statistical analysis of the conductance. For all scans, and resulting configuration, each 20 ps, we computed the conductance using a full quantum mechanical procedure based on the ab initio Gaussian embeddedcluster method. ${ }^{22}$ Due to computer time limitations, the conductance was computed for configurations with $S_{m} \leqslant 5$ restricting the quantum calculation to a nanocontact region formed by five atomic layers, describing the narrower (and most important, in terms of electronic transport) nanocontact section. This narrow layer is formed by the minimum crosssection layer (where $S_{m}$ is evaluated) and its two neighboring layers below and above. For different minimum cross-section regions, we have checked that the conductance values were not altered when including more layers in the calculations. In the present study the number of atoms involved in the conductance quantum calculations range between 20 and 50, de- pending on the constriction minimum cross-section size.

Typical evolution of the minimum cross-section $S_{m}$, and the corresponding conductance $G$, during the nanocontact breakage are shown in Fig. 1. The shapes of the curves reveal the existence of a strong correlation between conductance and the nanocontact neck section size, in agreement with previous numerical results for other materials., ${ }^{4,24} \mathrm{~A}$ striking fact is that for values $S_{m} \approx 1$ (defining a nanocontact of one-atom section) there are two different conductance values $\left(G / G_{0} \approx 2\right.$ and $\left.\approx 1\right)$. We have confirmed that this fact is explained in terms of the presence of two different atomic arrangements $^{7}$ at the last stage of the breaking process. On the one hand, the monomer contact configuration [see Fig. 1 (a)] provides conductance values of the order of $G / G_{0} \approx 2$, while on the other hand, the dimer contact configuration [see Fig. 1(b)] gives rise to conductances close to $G / G_{0} \approx 1$. This conductance bi-valuation, for the $S_{m} \approx 1$ case, shows that the orbital valence accommodates differently depending on the contact coordination and on the separation between the contact atom and its neighbors, a finding that confirms previous observations. $^{7,25}$ Also, we have noticed that when a neck section of two-atom contact lifts under stretching, a dimerchain contact is formed, giving rise to a conductance jump from a value greater than 3-1.2 [see Fig. 1(b)]. With increasing stretching (time in the figure), $G$ slowly decreases and then increases from $0.90 G_{0}$ to $1.05 G_{0}$. This occurs when the dimer-chain contact evolves from a position perpendicular to the (111) direction toward a parallel alignment with this direction (which is perpendicular to the supporting slabs). Such increase of $G$ before the rupture reproduces STM transport measurements, ${ }^{26}$ confirming the validity of our model, and reveals the possible improvement of electronic resonant conditions with strain, as predicted by previous numerical calculations. 5,26 Finally, the simulated conductance curve falls to 0 as the nanocontact breaks. In some cases (as those appearing in Fig. 1), these slow decays are different from those noticed in low voltage experimental curves. We at- 


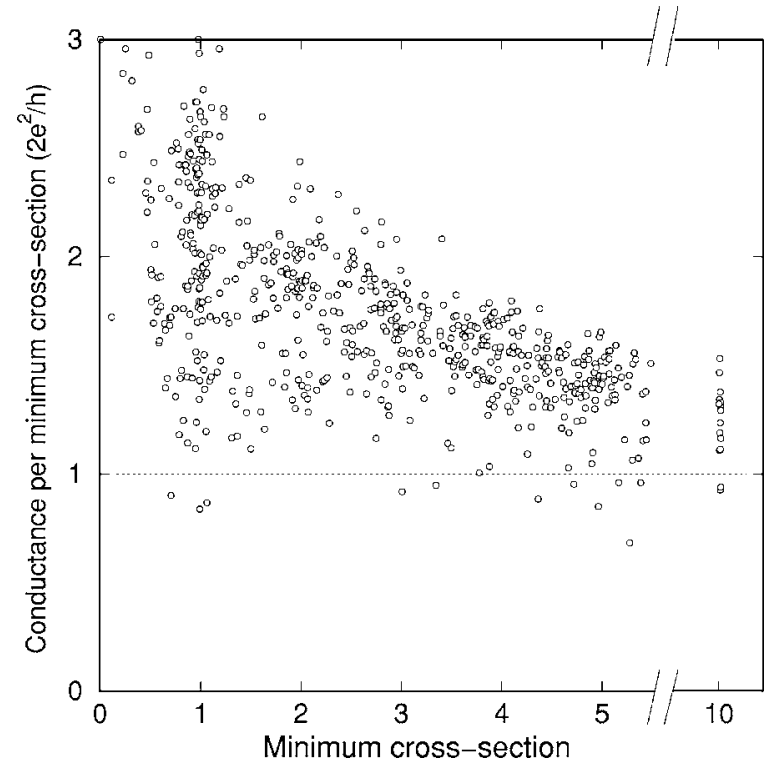

FIG. 2. Conductance per minimum cross-section $G_{a}$ as a function of the minimum cross-section $S_{m}$. The figure shows more than 800 points corresponding to 50 simulated aluminum nanocontact breakages. The dotted line is a guide to the eye and denotes the quantum conductance value $G_{0}$.

tribute such discrepancy to the higher time resolution that can be monitored by our molecular dynamics calculations.

For more than 800 aluminum nanocontact configurations (obtained from the evolution of 50 stretching sequences), by using full quantum procedures we have calculated the conductance per minimum cross-section $G_{a}=G / S_{m}$. This quantity is equivalent to the sum of the three channel transmitivities available for aluminum atoms at the narrowest neck section. Figure 2 depicts $G_{a}$ as a function of the minimum cross-section $S_{m}$. In spite of the data dispersion, the figure suggests that $G_{a}$ converges to a constant value as $S_{m}$ increases. We recall that the independence of $G_{a}$ on the contact size has been previously observed experimentally for gold nanocontacts, ${ }^{4}$ where it was observed that $G_{a}=G_{0}$, which is the maximum possible value for the atomic conductance of any monovalent material. ${ }^{4,16,24}$ For the aluminum case, Fig. 2 shows that for almost all configurations, $G_{a}$ results are larger than $G_{0}$, and converges to a value between $G_{0}$ and $1.5 G_{0}$. Then, the data suggest that aluminum nanocontacts are, per atom, better conductors than any monovalent metal wire.

As is usually done in experiments, $, 10,27,28$ we accumulated all $G$ traces and constructed the first computational aluminum conductance histogram (see Fig. 3, top). Note that, within the estimated errors (which is of the order of $G_{0} / 2$ for $S_{m}<5$, see the error bars in Fig. 4), such histogram shows a similar peak structure to that found in experiments. The most important result is that the conductance histogram shows peaks close to integer multiples of the conductance quantum. ${ }^{8,27,28}$ Here, the peak-to-valley ratio is smaller than in experimental conductance histograms, but this could be caused by the relatively small number of configurations used in our calculations. Also, the statistical analysis could improve by taking into account different orientations of the nanowire main axis. A more exhaust statistical treatment will
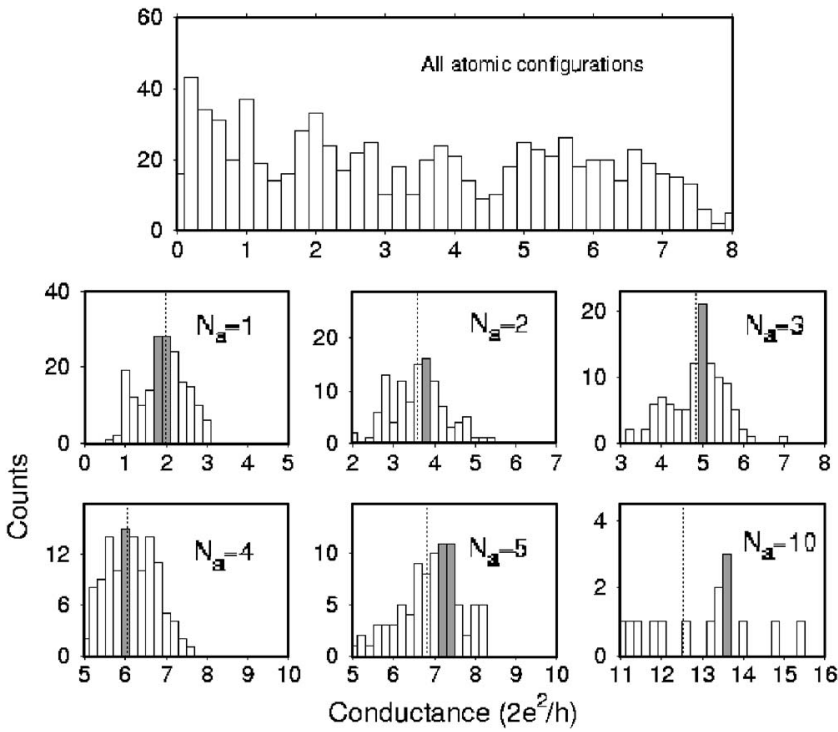

FIG. 3. Calculated conductance histogram for aluminum (top) using those points shown in Fig. 2. Below this histogram, we separately show the partial conductance histograms for those configurations corresponding to effective number of atoms $N_{a}=1,2,3,4,5$ and 10. Gray bins denote the maximum value of the conductance $G_{M}\left(N_{a}\right)$. Vertical dashed lines denote the computed average conductances $\left\langle G\left(N_{a}\right)\right\rangle$ for each depicted conductance distribution.

require a huge computational effort, but we believe that the appearance of a well defined peaked structure in the computational histogram of this trivalent metal is the most remarkable result of this work. Note that the presence of some conductance traces with slow conductance decays at the last stage of the nanowire breakage (as those shown in Fig. 1) modifies the conductance histogram below $G_{0}$, but not the

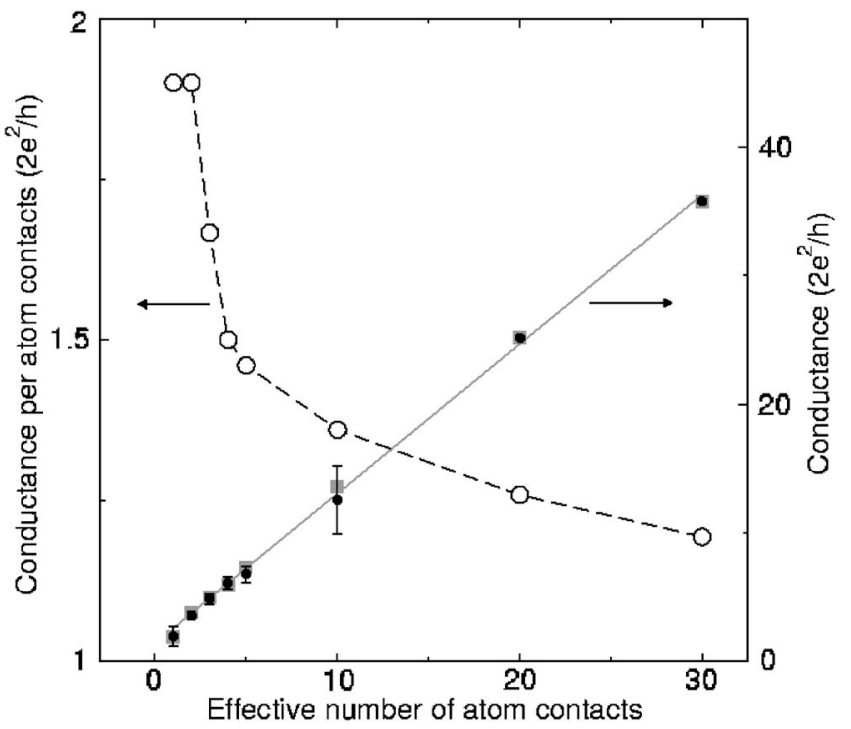

FIG. 4. Conductance per atom as a function of the effective number of atom contacts $N_{a}$ (open circles). Average conductance $\left\langle G\left(N_{a}\right)\right\rangle$ (black circles) and peak conductance $G_{M}\left(N_{a}\right)$ (gray squares) as a function of the effective number of atom contacts. Bars denote the standard error. Dashed line represents a linear fit to the $G_{M}\left(N_{a}\right)$ data. 
peaked structure at higher conductance values. We recall that a peaked structure has also been observed in minimum crosssection histograms, ${ }^{15,23}$ reflecting the existence of energetically favorable atomic configurations at the nanocontact neck.

Regarding experiments, the main advantage, when performing molecular dynamics simulations, is that we can separate the corresponding conductance contributions in nanocontact configuration families, grouping configurations that possess a similar number of atoms at the narrowest nanocontact cross section. In order to identify such configuration families, we use the integer label $N_{a}\left(N_{a}=1,2,3 \ldots\right)$ to describe the set of configurations with minimum crosssection $S_{m}$ comprised between $N_{a}-1 / 2$ and $N_{a}+1 / 2$. This label is equivalent to the effective number of atoms defining the narrowest nanocontact region. Partial conductance histograms concocted in the previous fashion are depicted in Fig. 3 and show that for all $N_{a}$ values, there corresponds a peak at a conductance value $G_{M}\left(N_{a}\right)$. For comparison, we also show in Fig. 3 the average conductance $\left\langle G\left(N_{a}\right)\right\rangle$ for each partial conductance histogram (see vertical dashed lines). Note that for the monoatomic contact case $\left(N_{a}=1\right)$, two conductance peaks appear at $G / G_{0}=1$ and $G / G_{0}=2$, which correspond, respectively, to the conductance contributions of the dimer chain [see inset of Fig. 1(b)], and the single-central one-atom [see inset of Fig. 1(a)] contact configurations. For $N_{a} \geqslant 2$ the average conductance $\left\langle G\left(N_{a}\right)\right\rangle$ is close to the peak position $G_{M}\left(N_{a}\right)$ indicating that conductance distributions are rather symmetrical around its maximum value. A very important conclusion regarding our calculations is that the first two peaks of the aluminum conductance histogram (centered around $G=G_{0}$ and $G=2 G_{0}$ ) exhibited by experiments are originated by the $N_{a}=1$ group, and correspond to dimer and monomer-like configurations, respectively. In addition, it is clear that the minimum cross-section histogram is unable by itself to provide such specific information, and therefore this kind of computational procedure requires further analysis.

Figure 4 plots the quantity $G_{M}\left(N_{a}\right) / N_{a}$ (open circles) as a function of the effective number of atom contacts $N_{a}$. In this figure, we have included two points corresponding to particular aluminum nanocontact configurations with $N_{a}=20$ and 30. The convergence of the atomic conductance to a constant value between 1 and 1.5 is now much more evident than in Fig. 2. Figure 4 also includes a plot of the conductance maximum $G_{M}\left(N_{a}\right)$ as a function of $N_{a}$ (gray squares, corresponding label figure appears at the right hand side). The good quality of a linear fit of the data $\left(\chi^{2}=0.9995\right)$ suggests that the conductance converges to a straight line (with a slope of $\left.1.16 G_{0}\right)$. Additionally, we plot the average conductance $\left\langle G\left(N_{a}\right)\right\rangle$ for each contact configuration family $N_{a}$ (small black circles in Fig. 4), and its corresponding standard error. Within the estimated error bars, the $\left\langle G\left(N_{a}\right)\right\rangle$ values reflect the same linear behavior observed for $G_{M}\left(N_{a}\right)$. Therefore it is evident that a linear relation between the conductance and the effective number of atom contacts $N_{a}$ can be established. A finding which is far to be obvious, ${ }^{19}$ mainly because at the quantum limit the backscattering process can be the determinant of the electronic transport features in many materials. For aluminum, platinum as well other multivalent nanocontacts, it has been evidenced that the transmission probability per channel can result much smaller than $1^{3}$, a fact that cannot be accounted for by any semiclassical approximation. ${ }^{17,18}$ Furthermore, semiclassical approximations based on the free electron model have a fundamental shortcoming in the face of the previous result: They only depend on geometry, and the Fermi wavelength is the only material dependent parameter, while backscattering due to both geometrical and electronic structure constraints is the main culprit for the appearance of ballistic resistance in nanomaterials. ${ }^{3,24}$ In our conductance calculations, the scattering phenomenon is implicitly described in the considered quantum methodology. The slope of the curve in Fig. 4 is now an electronic structure specific property that can be bundled into a peculiar ballistic resistivity defined by

$$
G=N_{a} / \rho_{b},
$$

where $N_{a}$ is dimensionless and $1 / \rho_{b}$ has the dimensions of conductance. Here, $\rho_{b}$ retains the value of $0.86 R_{0}$, where $R_{0}=1 / G_{0}=12907 \Omega$. In that sense the new ballistic resistivity is a result of a novel scaling behavior of the material conductance at the mesoscale. It is this scaling that promises to be an universal material independent property.

In our work, the notion of the area $A$, instead of $N_{a}$, is inadequate since any consideration defining it in a complex electronic structure of few atom contacts results speculative. Notwithstanding it is intuitive that a first order approximation for such an area should behave linearly with the effective number of atom contacts $N_{a}$.

Finally, one should expect that the described ballistic resistivity $\rho_{b}$ will also depend on thermodynamical variables, as the minimum cross-section histograms depend on temperature. ${ }^{15}$ Such properties, and the extension of this kind of study, on other chemical elements with different electronic structures, seem to conform to a field of a promising research activity, due to the obvious attractive applications of this knowledge in the emerging nanoelectronic industry.

We thank J. J. Sáenz for helpful discussions, and Cecalcula (Venezuela) for computer facilities. This work has been partially supported by the CSIC-IVIC researchers exchange program and the Spanish DGICyT (MEC) through Project No. BFM2003-01167/FISI.

\footnotetext{
${ }^{1} \mathrm{~A}$ review on the current status on metallic nanowires research is: N. Agraï, A. Levy-Yeyati, and J.-M. van Ruitenbeek, Phys. Rep. 377, 81 (2003).

${ }^{2}$ R. Landauer, Philos. Mag. 21, 863 (1970).
}

${ }^{3}$ E. Scheer, N. Agraït, J. C. Cuevas, A. Levy-Yeyati, B. Ludoph, A. Martin-Rodero, G. Rubio-Bollinger, J. M. van Ruitenbeek, and C. Urbina, Nature (London) 394, 154 (1998).

${ }^{4}$ V. Rodrigues, T. Fuhrer, and D. Ugarte, Phys. Rev. Lett. 85, 4124 
(2000).

${ }^{5}$ Y. J. Lee, M. Brandbyge, M. J. Puska, J. Taylor, K. Stokbro, and R. M. Nieminen, Phys. Rev. B 69, 125409 (2004).

${ }^{6}$ J. C. Cuevas, A. Levy-Yeyati, and A. Martín-Rodero, Phys. Rev. Lett. 80, 1066 (1998).

${ }^{7}$ P. Jelinek, R. Pérez, J. Ortega, and F. Flores, Phys. Rev. B 68, 085403 (2003).

${ }^{8}$ A. I. Yanson and J. M. van Ruitenbeek, Phys. Rev. Lett. 79, 2157 (1997).

${ }^{9}$ A. I. Yanson, I. K. Yanson, and J. M. van Ruitenbeek, Nature (London) 400, 144 (1999).

${ }^{10}$ E. Medina, M. Díaz, N. León, C. Guerrero, A. Hasmy, P. A. Serena, and J. L. Costa-Krämer, Phys. Rev. Lett. 91, 026802 (2003).

${ }^{11}$ N. Agraït, J. G. Rodrigo, and S. Vieira, Phys. Rev. B 47, R12345 (1993).

${ }^{12}$ L. Olesen, E. Laegsgaard, I. Stensgaard, F. Besenbacher, J. Schiotz, P. Stoltze, K. W. Jacobsen, and J. K. Norskov, Phys. Rev. Lett. 72, 2251 (1994).

${ }^{13}$ J. M. Krans, J. M. van Ruitenbeek, V. V. Fisun, I. K. Yanson, and L. J. de Jongh, Nature (London) 375, 767 (1995).

${ }^{14}$ J. L. Costa-Krämer, N. García, P. Gacía-Mochales, and P. A. Serena, Surf. Sci. 342, L1144 (1995); Erratum in Surf. Sci. 349, L138 (1996).

${ }^{15}$ A. Hasmy, E. Medina, and P. A. Serena, Phys. Rev. Lett. 86, 5574 (2001).
${ }^{16}$ A. I. Yanson, I. K. Yanson, and J. M. van Ruitenbeek, Phys. Rev. Lett. 87, 216805 (2001).

${ }^{17}$ Y. V. Sharvin, Zh. Eksp. Teor. Fiz. 48, 984 (1965) [Sov. Phys. JETP 21, 655 (1965)].

${ }^{18}$ J. A. Torres, J. I. Pascual, and J. J. Sáenz, Phys. Rev. B 49, 16581 (1994).

${ }^{19}$ A. M. Bratkovsky, A. P. Sutton, and T. N. Todorov, Phys. Rev. B 52, 5036 (1995).

${ }^{20}$ T. López-Ciudad, A. García-Martín, A. J. Caamaño, and J. J. Sáenz, Surf. Sci. 440, L887 (1999).

${ }^{21}$ Y. Mishin, D. Farkas, M. J. Mehl, and D. A. Papaconstantopoulos, Phys. Rev. B 59, 3393 (1999).

${ }^{22}$ J. J. Palacios A. J. Pérez-Jiménez, E. Louis, E. SanFabián, and J. A. Vergés, Phys. Rev. B 66, 035322 (2002).

${ }^{23}$ M. Dreher, F. Pauly, J. Heurich, J. C. Cuevas, E. Scheer, and P. Nielaba, Phys. Rev. B 72, 075435 (2005).

${ }^{24}$ A. Nakamura, M. Brandbyge, L. B. Hansen, and K. W. Jacobsen, Phys. Rev. Lett. 82, 1538 (1999).

${ }^{25}$ N. D. Lang, Phys. Rev. B 52, 5335 (1995).

${ }^{26}$ J. C. Cuevas, A. Levy-Yeyati, A. Martín-Rodero, G. R. Bollinger, C. Untiedt, and N. Agraït, Phys. Rev. Lett. 81, 2990 (1998).

${ }^{27}$ M. Díaz, J. L. Costa-Krämer, P. A. Serena, E. Medina, and A. Hasmy, Nanotechnology 12, 118 (2001).

${ }^{28}$ A. Halbritter, Sz. Csonka, O. Yu. Kolesnychenko, G. Mihály, O. I. Shklyarevskii, and H. van Kempen, Phys. Rev. B 65, 045413 (2002). 